\title{
Adicción a las Redes Sociales en Tiempos de Covid: Un Enfoque desde la Minería de Datos
}

\section{Addiction to Social Networks in times of Covid: an approach from data mining}

\author{
Felipe de Jesús Núñez Cárdenas ${ }^{a}$, Ana María Felipe-Redondo ${ }^{b}$, Mayte Guerrero-Ruiz ${ }^{c}$, \\ Abigail Rivera-Hernandez ${ }^{d}$, Victor T Tomas-Mariano ${ }^{e}$
}

\begin{abstract}
:
In current times of confinement of the population as a result of the COVID pandemic, adolescents have become more vulnerable in the use of different applications on the web, among the most used are social networks. The present project aims to know the degree of addiction to social networks of high school students of the Higher School of Huejutla in times of COVID, understanding this with the levels of high, moderate and without addiction, For this the algorithm was used Kmeans within the Clustering technique. This algorithm was modeled in the WEKA tool, the methodology used is the so-called CRISP-DM. Data collection was carried out with the application of the Addiction Risk Scale Test in Social Networks (ERA-RSI). The results obtained are shown in three different clusters, one oriented to discharge, the second to moderate and a last to the population without addiction.
\end{abstract}

Keywords:

ERA RSI test, Kmeans algorithm, Dataminig

Resumen:

En tiempos actuales del confinamiento de la población como consecuencia de la pandemia de COVID, ha vuelto a los adolescentes más vulnerables en el uso de diferentes aplicaciones en la web, dentro de los más utilizados se encuentran las redes sociales. El presente proyecto tiene como objetivo conocer el grado de adicción a las redes sociales de los alumnos de bachillerato de la Escuela Superior de Huejutla en tiempos de COVID, entendíendose ésta con los niveles de alta, moderada y sin adicción, Para ello se utilizó el algoritmo Kmeans dentro de la técnica de Agrupamiento. Este algoritmo fue modelado en la herramienta WEKA, la metodología empleada es la denominada CRISP-DM. La recogida de datos se llevó a cabo con la aplicación del Test Escala de Riesgos de Adicción en las Redes Sociales (ERA-RSI). Los resultados obtenidos se muestran en tres diferentes cluster, uno orientado a la alta, el segundo a la moderada y un último a la población sin adicción.

Palabras Clave:

Test ERA RSI , Algoritmo kmeans, Mineria de Datos

\section{Introducción}

En la Actualidad a causa de la Pandemia Mundial ocasionada por el COVID se han modificado muchos procesos con la finalidad de evitar el contagio de este virus. Algunos de ellos se han visto afectados de tal manera que se ha optado por realizarlos desde la casa. Uno de ellos es el Educativo el cual se lleva a cabo de manera virtual. Dentro de la Escuela Superior Huejutla no ha sido la excepción, sin embargo, esta cuarentena en el hogar ha ocasionado que se disponga de la tecnología para aminorar este aislamiento.

\footnotetext{
a Autor de Correspondencia, Profesor de Tiempo Completo, Universidad Autónoma del Estado de Hidalgo, Escuela Superior de Huejutla, ORCID: https://orcid.org/0000-0002-2462-3654, email: felipe_nunez@uaeh.edu.mx

${ }^{\mathrm{b}}$ Universidad Tecnológica de la Huasteca Hidalguense. ORCID: https://orcid.org/0000-0002-8579-6532 Email: ana.felipe@uthh.edu.mx

c Universidad Autónoma del Estado de Hidalgo, Escuela Superior de Huejutla, email: gu359600@uaeh.edu.mx

${ }^{d}$ Universidad Autónoma del Estado de Hidalgo, Escuela Superior de Huejutla, email: ri362160@uaeh.edu.mx

e Universidad Autónoma del Estado de Hidalgo, Escuela Superior de Huejutla, ORCID: https://orcid.org/0000-0001-6623-860X, email: victor_tomas@uaeh.edu.mx
} 
Este trabajo se enfoca sobre la utilización de herramientas de minería de datos aplicando una técnica de agrupamiento y el algoritmo k-means, para determinar el nivel de dependencia a las redes sociales en los alumnos de bachillerato de la Escuela Superior Huejutla, usando como herramienta de evaluación el test ERA RSI [1], el cual nos puede diagnosticar el nivel de dependencia o adicción a este recurso.

Por otro lado, la minería de datos es el conjunto de técnicas de análisis que nos ayuda a predecir y describir el comportamiento de los datos. También extraer patrones, tendencia y regularidades [2].

Bajo este contexto se pretende diagnosticar bajo estas técnicas, el grado de adicción de los alumnos a dichas plataformas.

\section{Adicción a las Redes Sociales}

Las redes sociales como Instagram, Facebook, Twitter y los sistemas de mensajería como WhatsApp son atractivos para los jóvenes porque su funcionamiento implica la interactividad inmediata. Su uso es de carácter social para comunicarse en un ámbito popular, pero esto sin un control educativo puede llegar a trastornar la realidad de algunos, sobre todo cuando hablamos de jóvenes adolescentes. La adicción a las redes sociales se vincula a la mayoría de jóvenes que tienen carencias emocionales y acaban supliéndolas mediante las redes sociales o perfiles irreales.

Su uso nació bajo principios positivos, pero el problema ocurre cuando se detecta que se dejen de lado el resto de las obligaciones propias de una vida social normal. (estudiar o trabajar, hacer deporte u otras aficiones, salir con amigos o relacionarse con la familia). Además, los últimos estudios han detectado que el abuso de las redes sociales provoca alejamiento del entorno de la vida real, provoca niveles altos de ansiedad, afecta a la autoestima y puede acabar causando la perdida de la capacidad de autocontrol [3].

Hay personas que son más vulnerables que otras, por lo que los perfiles de los adictos a las redes sociales son bastante determinados. Si bien la mayoría de redes sociales están disponibles para todo el mundo, solo una pequeña parte pueden presentar problemas de abuso y adicción. Generalmente, los adolescentes constituyen un potencial colectivo de riesgo porque son los que más se conectan a Internet, más horas pasan en línea y además, también están más familiarizados con las nuevas tecnologías actuales.

Existen ciertas características de personalidad o estados emocionales que tienden a aumentar la vulnerabilidad psicológica del adicto. El Dr. Fábregas, psiquiatra especialista en adicciones del centro CITA, explica que cuando hablamos de adicciones a redes sociales suele haber un problema subyacente que debe tratarse psicológicamente también. La timidez excesiva, baja autoestima, rechazo de la imagen corporal, depresión o hiperactividad son solo algunos ejemplos que pueden manifestarse tras la adicción.

En estos casos el abuso de Internet es como una cortina de humo que evidencia la existencia de un fuego para controlar, un problema de personalidad o un trastorno mental, que es lo que habría que abordar en realidad. Hay otros casos en los que se trata a personas que muestran una insatisfacción personal con su vida y que intentan ocultar esas carencias familiares o sociales. En estos casos Internet o las redes sociales actúan como una prótesis tecnológica que facilita el escape de la vida real, aunque a largo plazo tenga consecuencias negativas [4].

\section{Agrupamiento: Algoritmo Kmeans}

Los algoritmos de clustering son considerados de aprendizaje no supervisado. Este tipo de algoritmos de aprendizaje no supervisado busca patrones en los datos sin tener una predicción específica como objetivo (no hay variable dependiente). En lugar de tener una salida, los datos solo tienen una entrada que serían las múltiples variables que describen los datos. K-means necesita como dato de entrada el número de grupos en los que vamos a segmentar la población. A partir de este número $\mathrm{k}$ de clusters, el algoritmo coloca primero $\mathrm{k}$ puntos aleatorios (centroides). Luego asigna a cualquiera de esos puntos todas las muestras con las distancias más pequeñas.

A continuación, el punto se desplaza a la media de las muestras más cercanas. Esto generará una nueva asignación de muestras, ya que algunas muestras están ahora más cerca de otro centroide. Este proceso se repite de forma iterativa y los grupos se van ajustando hasta que la asignación no cambia más moviendo los puntos. Este resultado final representa el ajuste que maximiza la distancia entre los distintos grupos y minimiza la distancia intragrupo.

El algoritmo k-Means sigue los siguientes pasos:

- Inicialización: se elige la localización de los centroides de los $\mathrm{K}$ grupos aleatoriamente

- Asignación: se asigna cada dato al centroide más cercano

- Actualización: se actualiza la posición del centroide a la media aritmética de las posiciones de los datos asignados al grupo.

Los pasos de asignación y actualización se siguen interactivamente hasta que no haya más cambios [5].

\section{Adicción a las Redes Sociales Aplicando Minería de Datos}


En el año 2015 Roció Leticia Cortes Campos, Alfredo Zapata Gómez, Víctor Hugo Menéndez y Pedro José Canto Herrera en su trabajo de investigación titulado el estudio de los hábitos de conexión en redes sociales virtuales, para ello utilizaron la minería de datos, su propósito fue presentar una visión general de los hábitos de conexión a las redes sociales virtuales entre los estudiantes de pregrado. La información se obtuvo por medio de un cuestionario, aplicando a 458 alumnos de nivel licenciatura de la universidad Autónoma de Yucatán, México. El análisis y la interpretación se realizaron utilizando técnicas de minería de datos, mediante las cuales se obtuvieron grupos de estudiantes definidos por el género, la frecuencia de conexión y las redes sociales que consultan. También se extrajeron reglas de asociación que sugieren importantes condiciones socioeconómicas y laborales, sobre todo con respecto a los lugares y a los dispositivos utilizados por los estudiantes el momento de conectarse. [6]

Por otro lado, en el año 2015 Elena Asensio Blasco, en su trabajo de investigación titulado Aplicación de minería de datos en redes sociales/web, en el cual se pudo obtener información de las redes sociales, como Twitter. Su análisis correcto proporciona un valor adicional a la recuperación de información. Se basa en la realización de una aplicación que gestione y obtenga datos sobre el uso de los lenguajes oficiales de las comunidades autonómicas en Twitter. [7]

\section{Metodología}

El proceso del presente trabajo se llevó a cabo bajo la metodología CRISP-DM ya que contempla el ciclo de vida de un Proyecto de minería de datos [8].

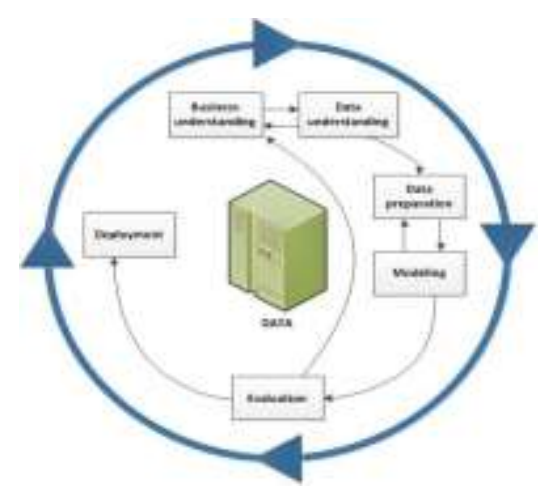

figura 1 Ciclo de Vida de un Proyecto de Minería de Datos, metodología CRISP -DM

\section{Desarrollo}

\section{Compresión del Caso de Estudio}

La Escuela Superior de Huejutla de la Universidad Autónoma del Estado de Hidalgo actualmente cuenta con los programas educativos de, Ciencias Computacionales, Derecho, Administración, Medicina y Enfermería en el nivel superior, además de ofertar el bachillerato General, es en esta sección donde se aplicó este proyecto. En promedio cuenta con una matrícula de 550 alumnos.

A partir de marzo del año 2020 y como consecuencia de la Pandemia del COVID 19, se tomó la determinación que las clases se llevaran a cabo de manera virtual por medio de plataformas tecnológicas. Por lo que el contexto se sitúa en educación a distancia bajo esta primicia. Este nivel de estudios de la ESH tiene dos horarios uno matutino y uno verpertino en donde los horarios de atención son de 7 am - 14:00 para el matutino y de 12:00 pm a 19:00 pm para el verpertino.

\section{Entendimiento de los Datos}

Como instrumento para la recolección de la información fue la encuesta de Escala de riesgo de adicciónadolescente a las redes sociales e internet (ERA-RSI) [9], el cual consta de 29 ítems, dicha encuesta se elaboró en Google Forms y se difundió por las redes sociales.

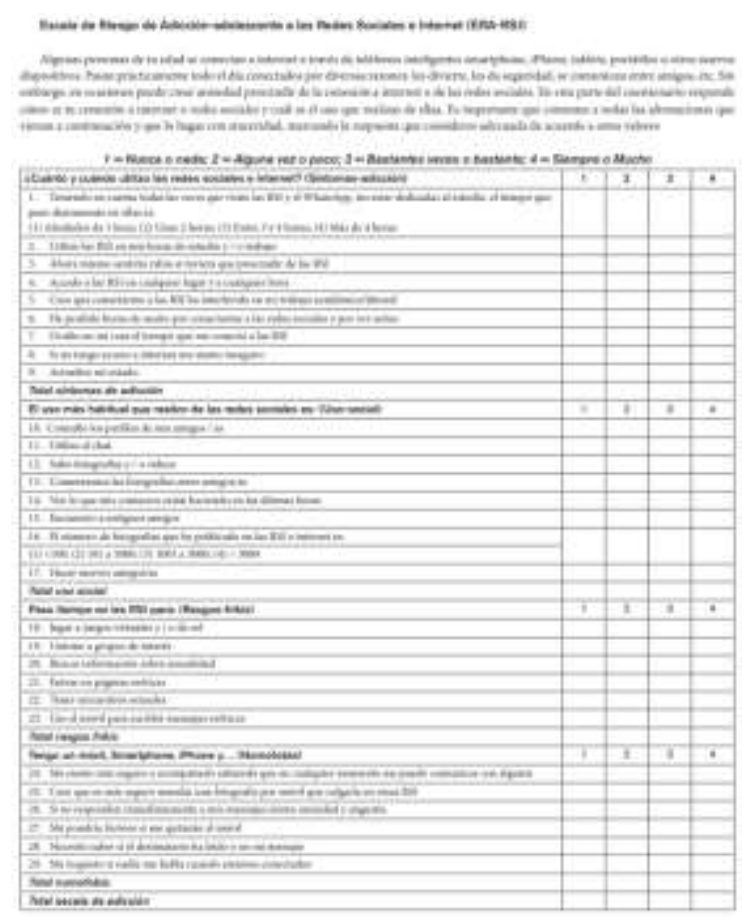

figura 2 Encuesta ERA RSI

Esta encuesta muestra como resultado tres niveles de adicción: Alta, Moderada y Sin Adicción. Y se trabaja bajo cuatro esquemas de determinación sobre estos niveles, es importante comentar que en el uso del instrumento de la encuesta ERA-RSI Podemos encontrar o se agrupan los idem en cuatro factores que originan el riesgo, estos 
son síntomas-adicción, uso-social, rasgos-frikis y nomofobia.

El factor síntomas-adicción checa conductas de adicción a sustancias no toxicas, el factor uso-social evalúa la socialización virtual de un adolescente, el factor rasgosfrikis recoge aspectos propios como reunirse con personas afines a algo y el factor nomofobia están relacionados a la ansiedad y control del uso del móvil [2].

\section{Preparación de los Datos}

Una vez obtenido los resultados de la encuesta, se preparan los datos iniciales para poder usarlos, para lo cual se puede utilizar la aplicación de Bloc de notas para su edición y se guarda el archivo con la extensión .arff, para así utilizarlos en la herramienta de explotación Weka.

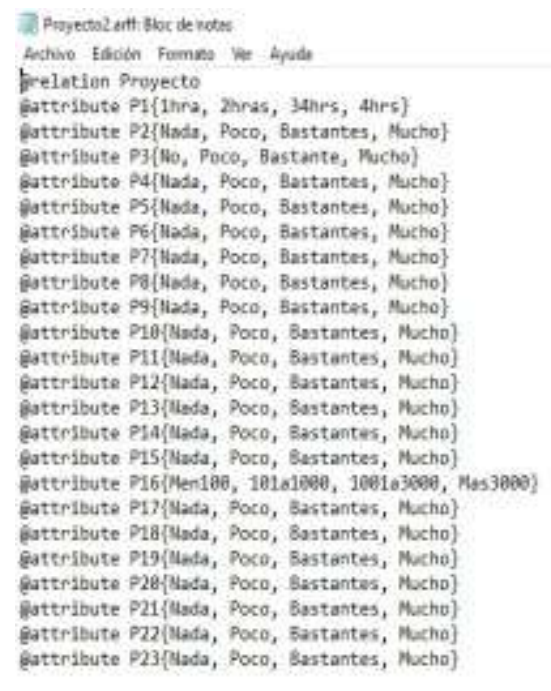

figura 3 preparación de datos en archivo .arff

\section{Modelado}

En esta etapa, se aplicó la técnica descriptiva de minería de datos, aplicando la herramienta Weka. Dentro de esta herramienta se utilizó la técnica de Agrupamiento en donde se optó por la creación de tres cluster, esto debido a la clasificación de la encuesta en sus tres niveles de adicción: alta, moderada y sin adicción. El algoritmo utilizado para tal fin es el Simple Kmeans.

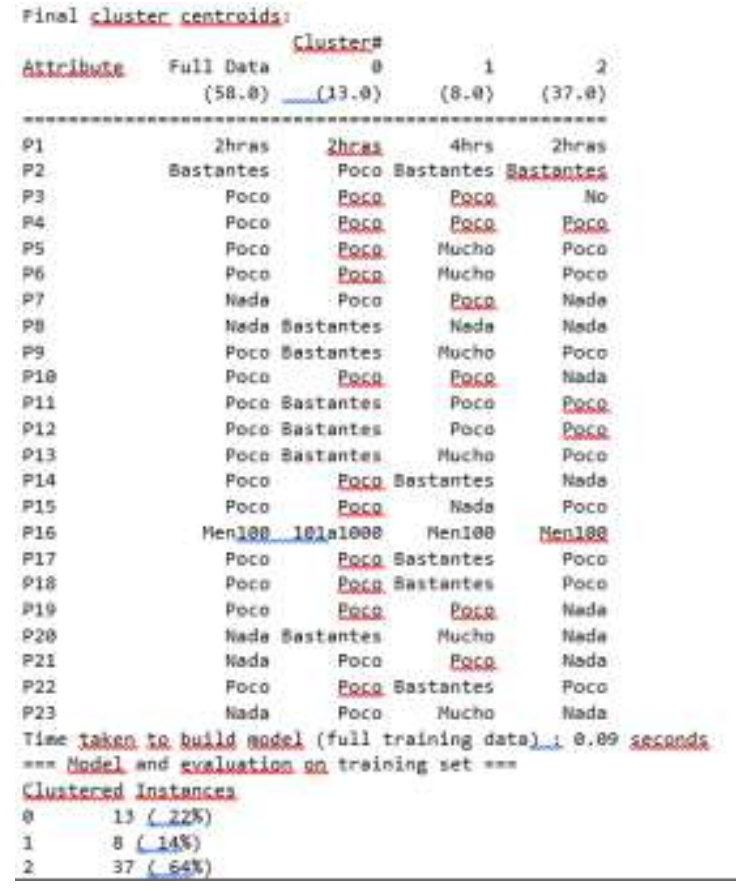

figura 4 Resultados del Modelo Ejecutado

\section{Resultados y Discusion}

Para la elaboración del modelo K-Means, con los 58 registros que corresponden al total de alumnos que contestaron la encuesta, los resultados que se obtuvieron fueron a través de tres cluster:

- $\quad$ Cluster 0: Representa una adicción moderada a las redes sociales que obtuvieron 13 de 58 que es equivalente a $22 \%$.

- Cluster 1: Representa una adicción alta a las redes sociales que obtuvieron 8 de 58 que es equivalente a $14 \%$.

- $\quad$ Cluster 2: Representa no tener una adicción a las redes sociales que obtuvieron 37 de 58 que es equivalente a $64 \%$.

las principales variables con que se obtuvieron estos resultados son el tiempo de uso, el acompañamiento virtual, la frecuencia de conexión, consulta de perfiles de amigos o conocidos, el uso más habitual para usar las redes sociales, entre otras, todas ellas referenciadas en los cuatro apartados del instrumento ERA -RSI. Podemos visualizar estas características en la figura 4.

\begin{tabular}{|c|cc|}
\hline CUUSTER & POBLACION & $\% \%$ \\
0 & 13 & $22 \%$ \\
\hline 1 & 8 & $14 \%$ \\
\hline 2 & 37 & $84 \%$ \\
\hline TOTAL & 58 & $100 \%$ \\
\hline
\end{tabular}

Tabla 1 resultados a nivel de cluster del modelado 
Es importante comentar que este Proyecto solo describe el indicador total de la adicción a las redes sociales sin adentrase a los diferentes factores síntomas-adicción, uso-social, rasgos-frikis y nomofobia. Con la intención de poder concluir un grado de adicción dentro de los diferentes grupos.

\section{Conclusiones y Trabajos Futuros}

Como conclusión de la aplicación del cuestionario ERARSI se obtuvo información importante sobre alumnos de bachillerato de la UAEH ESH, se aplicó el algoritmo KMeans para la agrupación de los datos, se eligieron 3 cluster, debido a que el ERA-RSI consta de 3 posibles diagnósticos que pueden dar de acuerdo con el puntaje obtenido en la evaluación. En base a los mismo Podemos decir que el $64 \%$ de la población no representa ningún síntoma de adicción a las redes sociales, mientras que el $22 \%$ presenta una adicción moderada a las mismas y el $14 \%$ presenta un alto grado de adicción a las redes sociales. Podemos a través de estos resultados el cual arroja la atención al 36\% de la población estudiantil de bachillerato implementar a través de técnicas de asociación y algoritmos como A priori encontrar correlaciones entre el desempeño académico y estos grados de adicción encontrados.

\section{Referencias}

[1] Florez, B. R., González, R. M., \& López, M. B. Valoración del riesgo de adicción a redes sociales e internet en adolescentes de la zona básica de salud de Mondéjar. CONDUCTAS DE RIESGO, 119.

[2] Orallo, J. H., Quintana, M. J. R., \& Ramírez, C. F. (2004). Introducción a la Minería de Datos. Pearson Educación.

[3] Cajas Ixco de Puluc, P. J. (2018). Experiencias psicológicas en el uso de la tecnología en adolescentes, estudio realizado en el Instituto Adrián Zapata durante el año 2017 (Doctoral dissertation, Universidad de San Carlos de Guatemala).

[4] Josep, D. M. (s.f.). ADICCIÓN A LAS REDES SOCIALES. Obtenido de https://www.clinicascita.com/adiccion-redes-sociales/

[5] Duck2. (9 de Enero de 2019). K-Means Clustering: Agrupamiento con Minería de datos. Obtenido de https://estrategiastrading.com/k-means/

[6] Rocío Leticia Cortes Campos, A. Z. (2015). el estudio de los hábitos de conexión en las redes sociales virtuales, por medio de la mineria de datos. ISSN, 99-114

[7] Blasco, E. A. (2015). Aplicación de técnicas de minería de datos en redes sociales/web. Valencia.
[8 ]Goicochea, A. (11 de Agosto de 2009). CRISP-DM, Una metodología para proyectos de Minería de Datos. Obtenido de Tecnologías de la Información y Estrategia: https://anibalgoicochea.com

[9] Peris, M., Maganto, C., \& Garaigordobil, M. (2018). Escala de riesgo de adicción-adolescente a las redes sociales e internet: fiabilidad y validez (ERA-RSI). 